\title{
Communications
}

\section{Chambre médicale ordinaire}

Entrée en vigueur des décisions de la Chambre médicale ordinaire du 28 mai 2009

Aucune demande de votation générale n'ayant été déposée durant le délai statutaire de 60 jours après la publication des décisions de la Chambre médicale ordinaire du 28 mai 2009 dans le Bulletin des médecins suisses (BMS N ${ }^{\circ} 28 / 29$ du 8 juillet 2009), celles-ci sont entrées en vigueur le 6 septembre 2009.

\section{Swiss Clinical Trial Organisation (SCTO)}

\section{Stärkung der klinischen Forschung in der Schweiz}

Mit einer gemeinsamen Initiative haben der Schweizerische Nationalfonds (SNF) und die Schweizerische Akademie der Medizinischen Wissenschaften (SAMW) die Voraussetzungen zur Gründung der Swiss Clinical Trial Organisation (SCTO) geschaffen. Die Aufgabe der SCTO als Dachorganisation der klinischen Forschung ist die Koordination und Harmonisierung der vielfältigen Aktivitäten von Studienzentren, die Vereinheitlichung der Qualitätsstandards sowie die Aus- und Weiterbildung von klinisch Forschenden.

$\mathrm{Zu}$ den Gründungsmitgliedern gehören die sechs Spitäler mit den vom SNF unterstützten klinischen Studienzentren (Clinical Trial Units in Basel, Bern, Genf, Lausanne, St. Gallen und Zürich) sowie die Medizinischen Fakultäten und die SAMW. Erster Präsident der SCTO ist Prof. Peter Meier-Abt, Vizepräsident der SAMW.

Ziele sind

- eine qualitativ hochstehende und national harmonisierte klinische Forschungskultur, einschliesslich der dafür notwendigen Weiter- und Fortbildungsangebote;

- die Bildung eines nationalen Netzwerkes, insbesondere zwischen einzelnen Studienzentren, der Akademie, der Industrie und den Behörden;

- die Einbindung der nationalen klinischen Forschung in internationale Netzwerke;

- günstige regulatorische Rahmenbedingungen für die klinische Forschung.

Weitere Informationen unter www.scto.ch

\section{Société Suisse \\ de Médecine nucléaire (SSMN)}

\section{Prix Bernard Delaloye}

Ce prix, doté d'un montant de Fr. 10000.-, sera décerné la troisième fois lors de la prochaine assemblée annuelle de la Société Suisse de Médecine nucléaire qui se tiendra du 3 au 5 juin 2010 à Lugano.

Les candidats sont priés de faire parvenir leurs travaux effectués dans le domaine de la Médecine nucléaire (recherche clinique ou de base) auprès d'un institut domicilié en Suisse ou par des chercheurs suisses à l'étranger et dont les résultats ont été publiés ou acceptés pour publication après le 1.1.2007, au Secrétariat de la SSMN, par courrier électronique, à Madame Irène Posner: irene.posner@kssg.ch, Médecine nucléaire, Hôpital cantonal, 9007 Staint-Gall. Dernier délai: 31 décembre 2009.

\section{International Foundation for Research in Paraplegia (IRP)}

Research grants in the field of Basic and Clinical Research on CNS Regeneration / Neuroprotection and Functional Restoration

With special emphasis on the spinal cord. Funds for one or two years will be allocated on the basis of scientific quality and relevance to paraplegia for research proposals studying mechanisms of injury and repair, as well as long term recovery. Applications by start up groups are welcome.

IRP, Geneva will also award one fellowship for up to two years to support post-doctoral research in paraplegia. Applications will be reviewed by an international panel of basic and clinical neuroscientists.

Deadline for applications: October 1, 2009.

Please use the corresponding online form to submit your application: www.irp.ch/grants. For more information please contact: info@ irp.ch

\section{Prix Galien Suisse}

\section{Orencia $^{\circledR}$ ausgezeichnet}

Medical Tribune hat zum achten Mal den Prix Galien Suisse 2009 verliehen. Der Gewinner des nationalen Preises qualifiziert sich für den internationalen Prix Galien, den «Nobelpreis für Pharmazie». Ziel des Preises ist die Würdigung der pharmakologischen Forschung im Dienste der Entwicklung neuer und innovativer Arzneimittel und Diagnostika. Der Gewinner ist Orencia ${ }^{\circledR}$ der Firma Bristol-Myers Squibb. Das Medikament ist ein biotechnologisch hergestelltes Eiweiss mit einem neuen Wirkmechanismus bei der Therapie der Rheumatoiden Arthritis.

\section{Bilddatenbank Medizin}

Die medizinische Web 2.0-Bilddatenbank auf www.medizinwelten.de hat den Bestand an medizinischem Bildmaterial vergrössert. Durch eine Kooperation mit der Inneren Abteilung des Ortenau Klinikums Achern hat sich der Fundus um mehr als 600 medizinische Bilder aus allen Bereichen der Inneren Medizin vergrössert. Thematischer Schwerpunkt sind neben der Kardiologie, Pulmologie und Rheumatologie insbesondere die Bereiche Angiologie und Gastroenterologie. medizinwelten ist auch im Internet-Netzwerk «Twitter« zu finden.

medizinwelten ist ein Internetangebot zum Mitmachen und erlaubt es Ärzten und medizinischen Fachkreisen, sowohl medizinisches Bildmaterial herunterzuladen als auch eigene Bilder einzustellen. Die Bilder können kostenlos und lizenzfrei verwendet werden, zum Beispiel für Vorträge oder eigene Publikationen.

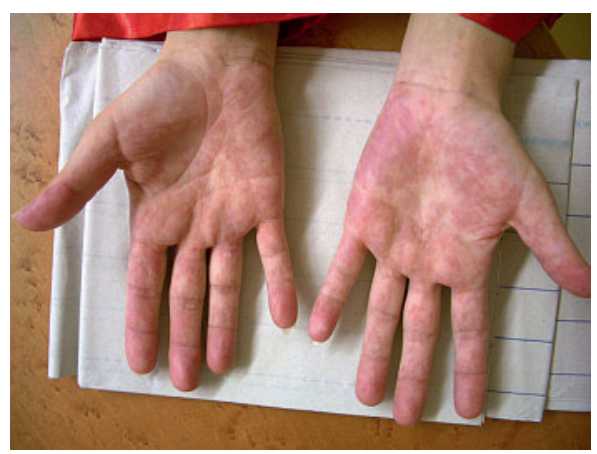

Palmarerythem bei einer Patientin mit systemischem Lupus erythematodes (SLE).

Quelle: medizinwelten. de; Dr. R. Feik, Klinikum Achern 